\title{
Characterization of the Chirp of Silicon Optical Modulators
}

\author{
Sheng Liu ${ }^{(1)}$, David J. Thomson ${ }^{(2)}$, Frederic Y. Gardes ${ }^{(2)}$, Jean-Marc Fedeli ${ }^{(3)}$, \\ Periklis Petropoulos ${ }^{(1)}$ and Graham T. Reed ${ }^{(2)}$ \\ (1) Optoelectronics Research Centre, University of Southampton, UK, shl@orc.soton.ac.uk \\ (2) School of Electronics and Computer Science, University of Southampton, UK. \\ (3) CEA, LETI, Minatec Campus, Grenoble, France.
}

\begin{abstract}
The chirp of a fast silicon optical modulator is characterized in this paper. Two diagnostic methods, frequency resolved optical gating and optical modulation analysis, are employed respectively. We demonstrate that the output of silicon optical modulators have very small intrinsic chirp across the modulated pulses.
\end{abstract}

\section{Introduction}

Optical modulators formed in silicon are the key milestone to achieving low cost optical communications. Devices based in silicon offer the attractive prospect of integration with complementary metal-oxide-semiconductor (CMOS) electronics on the same substrate and operation at longer wavelengths. Over the previous decade dramatic improvements in device performances have been demonstrated worldwide with most current devices achieving data modulation of at least $10 \mathrm{Gbit} / \mathrm{s}$. The devices used in this work have achieved modulation at $10 \mathrm{Gbit} / \mathrm{s}^{1}, 40 \mathrm{Gbit} / \mathrm{s}^{2}$ and 50 $\mathrm{Gbit} / \mathrm{s}^{3}$. While there has been a significant amount of effort in improving device performances in terms of speed, modulation depth, size, power consumption etc., there has been little consideration of the chirp from silicon optical modulators. In this work we aim to firstly understand the causes of chirp in silicon optical modulators using a theoretical approach and then both theoretically and experimentally characterize the chirp produced from example devices.

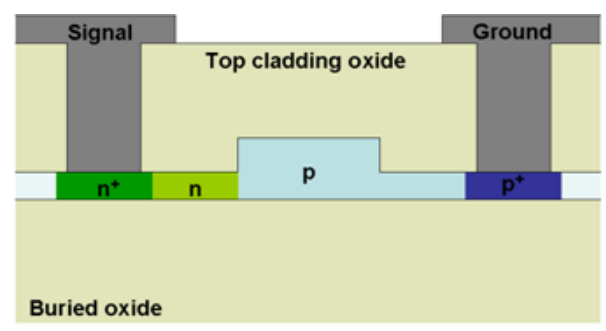

Fig. 1: Cross-sectional diagram of phase modulator

\section{Silicon optical modulator}

The phase modulator used in this analysis is as has been previously reported ${ }^{1-3}$. The cross sectional diagram is shown in Fig.1. The device is formed in rib waveguides of height $220 \mathrm{~nm}$, width $400 \mathrm{~nm}$ and slab height $100 \mathrm{~nm}$. The device operates via the plasma dispersion effect using carrier depletion to electrically control the density of free electrons and holes in the waveguide. A pn junction is therefore formed in the vicinity of the waveguide. Coplanar waveguide electrodes contact to highly doped $p$ and $n$ type regions to drive the device at high speed. The phase modulators are incorporated into asymmetric Mach-Zehnder Interferometers (MZI) to convert from phase modulation to intensity modulation.

\section{Chirp in silicon optical modulators}

The changing concentration of free electrons and holes in the material changes both the real and imaginary parts of the refractive index ${ }^{4}$. As a result, with the MZI modulator operating in dual drive configuration, the optical power from each arm will be changing with time. As one arm is 'on' when the other is 'off' the balance of optical power from the two arms will shift slightly from one to the other during modulation. The output phase will tend towards the phase of the arm with the greatest optical output power and will therefore also change with time. This means that in theory, unlike $\mathrm{LiNbO}_{3}$ modulators, it is not possible to achieve zero chirp from a silicon MZI optical modulator in dual drive.

A theoretical model representing the silicon MZI optical modulator has been developed to assess the chirp produced. A $20 \mathrm{Gbit} / \mathrm{s}$ PRBS data stream has been generated and filtered to match the shape of the experimental data stream from the optical modulator. The model then scales and applies complementary PRBS data streams to the two MZI arms. The scaling defines that amount of phase modulation produced by each arm, and is set to match the output extinction ratio of the experimental device. The normalised output power from the modeled device against time is shown along with the theoretical shift in optical carrier frequency in Fig.2. It can be seen that the peak frequency shift is approximately $0.5 \mathrm{GHz}$. 


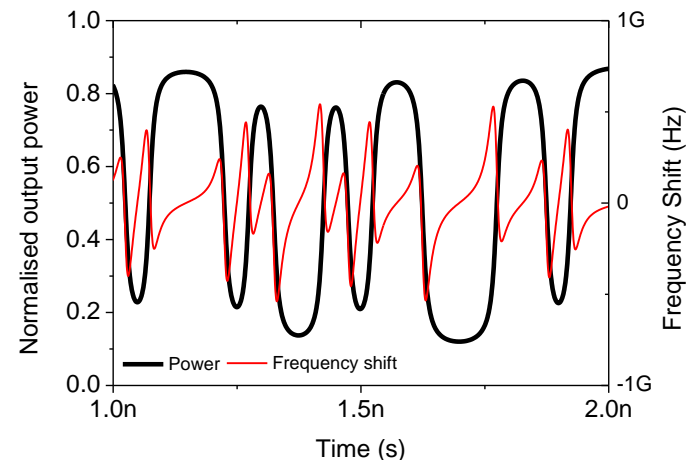

Fig. 2: Simulated modulator normalized output magnitude and frequency shift

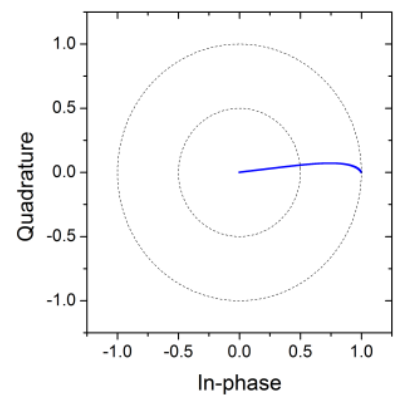

Fig. 3: Simulated constellation diagram

Fig. 3 shows the simulated output magnitude during the transition between the 1 and 0 data levels. The minimum is set to $(0,0)$ and data normalized so that the maximum occurs at $(1,0)$. A straight transition between the minimum and the maximum indicates zero chirp. Only a slight deviation from this condition can be seen in Fig. 3 therefore demonstrating a low level of chirp.

\section{Experiments}

Two kinds of diagnostic tools, linear-frequency resolved optical gating (I-FROG) and optical modulation analysis, were employed to measure the chirp at the silicon modulator output.

We first characterized the modulator output using I-FROG. FROG is a well-known technique for fully characterizing (in both phase and amplitude) short optical pulses ${ }^{5}$. In the I-FROG variation of the technique, optical gating is achieved through the use of an electro-optic modulator (EOM), making it particularly suited for low-power telecoms signals ${ }^{6}$.

The experimental setup for the I-FROG

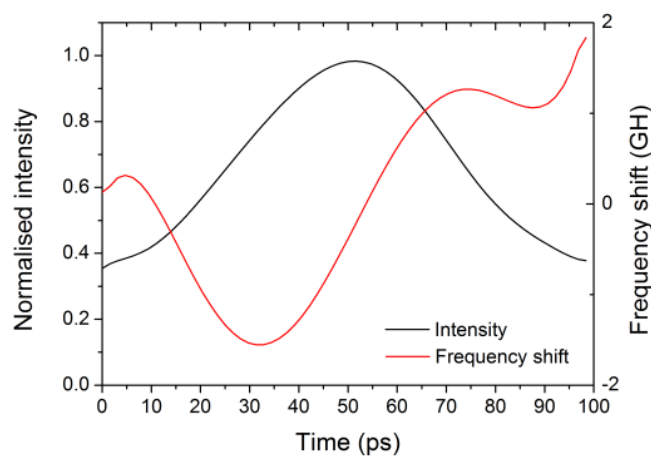

Fig. 5: Chirp and intensity profiles of the silicon modulator output

measurement is shown in Fig. 4. A CW laser at $1549.5 \mathrm{~nm}$ was amplified to $20 \mathrm{dBm}$ and launched into the dual-drive silicon modulator. The silicon modulator was driven by a pattern generator which operated at $20 \mathrm{Gbit} / \mathrm{s}$ and generated a non-return-to-zero on-off keying pattern of 0101 . The modulated output was amplified to $3.5 \mathrm{dBm}$ and sent to the EOM after polarization optimization. The gate pulses were generated by a gain switched laser diode ( 7 ps full width at half maximum) and sent to a variable delay line and a constant gain optical amplifier, which was to provide an appropriate power level for the receiving photodiode $(20 \mathrm{GHz}$ bandwidth). The electrical pulse signal from the photodiode was used as the drive signal for the EOM. This configuration allowed the optical signal at the input of the EOM to be gated by the electrical pulse, while gradual sweeping of the variable delay line produced the crosscorrelation of the two signals at the output of the EOM. The frequency resolved cross-correlation signal (spectrogram) was captured through successive measurements on an OSA. An iterative retrieval algorithm was then applied for reconstructing the intensity and phase profiles of the pulse from the I-FROG spectrogram trace. (Note that use of a short pattern on the pattern generator ensured that the spectrogram of constant bit values was captured on the OSA, rather than an average of zero and one bits). The retrieved intensity and phase profiles of the silicon modulator output are shown in Fig.5. The modulated signal had a pulse width of $\sim 50$ ps and its chirp was less than $3 \mathrm{GHz}$ across the

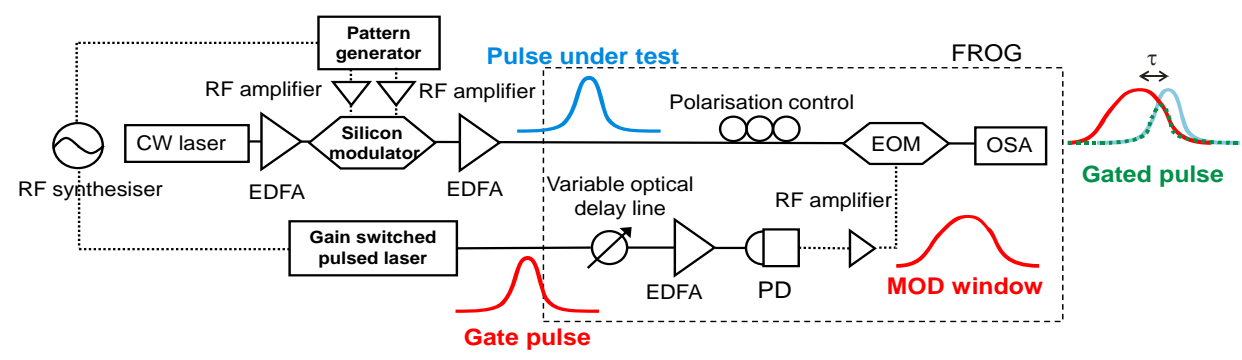

Fig. 4: Experimental setup of FROG measurement. EOM: electro-optic modulator, EDFA: Erbium doped fiber amplifier, PD: photodiode, OSA: optical spectrum analyser. 


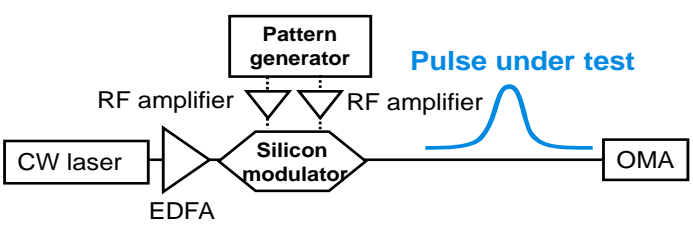

Fig. 6: Experimental setup of OMA measurement pulse. This amount of chirp is similar to that arising from a transform-limited signal after just a length of $100 \mathrm{~m}$ of SMF. This is not very much longer than the estimated total fibre length in the I-FROG setup. Therefore, it is expected that the chirp produced by the dual drive MZI silicon modulator was much smaller than this, in accordance to the prediction from the simulation. In the second set of experiments, the output of the silicon modulator was characterised using an optical modulation analyser (OMA - Agilent $\mathrm{N} 4391 \mathrm{~A}$ ), used in conjunction with an 80GSa/s real-time oscilloscope (Agilent DSO-X 93204A). An OMA is capable of analysing a complex signal and offering constellation analysis. Similar to the previous experiment, a CW laser operating at $1545.4 \mathrm{~nm}$ was modulated by the silicon modulator which was driven by a pattern generator working at $20 \mathrm{Gbit} / \mathrm{s}$ and with a pattern of 0101 (chosen for easier analysis of the data - see Fig.6). Figure 7 shows the $\mathrm{I}-\mathrm{Q}$ constellation diagram based on 2048 samples, as measured with the OMA. Due to a poor signal-to-noise ratio the transitions between the 1 and 0 levels appear blurred in the measured constellation diagram. Nevertheless, a very small chirp is confirmed, commensurate with the theoretical prediction (also shown in Fig.7). Note that the much simpler experimental setup of the OMA measurement allows the signal under test to be measured with much less residual chromatic dispersion, compared to the case of IFROG. Figure 8 shows the averaged intensity profile, as well as the chirp profile during the transition. Except for the pulse tails (where the optical power, and consequently the confidence

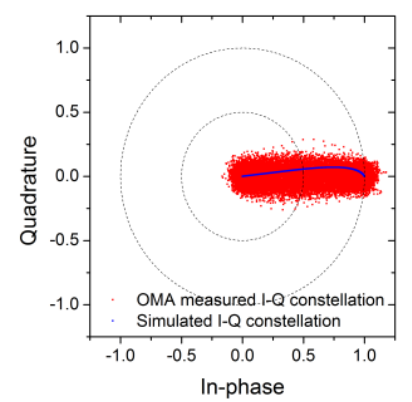

Fig. 7: Constellation diagram measured with OMA. (The mean of the distribution of the amplitude minimum is set to $(0,0)$ and the data normalized so that the maximum occurs at $(1,0)$.)

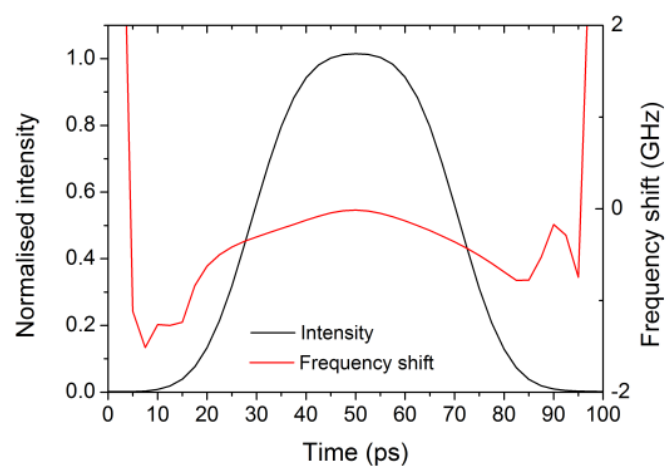

Fig. 8: Chirp and intensity profiles of the silicon modulator output measured with OMA. The minimum and maximum of the intensity magnitudes are nomalised to 0 and 1 respectively.

in the measured values, is low), the measured chirp was under $1 \mathrm{GHz}$ across the pulse.

\section{Conclusions}

In this paper we have theoretically and experimentally analysed the chirp from a dualdrive $\mathrm{MZI}$ silicon optical modulator. The simulation predicts that the peak chirp produced is approximately $500 \mathrm{MHz}$ under the conditions that the modulator was driven experimentally. The chirp measured by I-FROG was just that expected from $100 \mathrm{~m}$ of SMF from a chirp-free modulation source and therefore the chirp produced by the silicon optical modulator was small. A low level of chirp was also confirmed by OMA measurements where the transition showed no significant deviation from a straight, chirp-free case. These results highlight the suitability of silicon optical modulators for use in high-speed transmission systems, as they demonstrate that the link design needs not to be compromised in order to benefit from the advantages offered by silicon.

\section{Acknowledgements}

The research leading to these results has received funding from the European Community's Seventh Framework Programme (FP7/2007-2013) under grant agreement no. 224312 HELIOS and from the EPSRC in the UK to support the UK Silicon Photonics Project and the Photonics Hyperhighway (EP/I01196X). D.J.T would like to acknowledge $H$. Porte for discussions.

\section{References}

[1] D. J. Thomson et al., Opt. Express, 18(18), , 2010.

[2] D. J. Thomson et al., Opt. Express, 19(12), , 2011.

[3] D. J. Thomson et al.,IEEE PTL, 24(4), 2012.

[4] G. T. Reed et al., Nature Photonics, 4, 2010.

[5] R. Trebino, Frequency-Resolved Optical Gating, Springer (2005).

[6] K.T. Vu et al., IEEE PTL, 20(7), 2008. 